\title{
Flow Complexity Estimation in Dysfunctional Arteriovenous Dialysis Fistulas using Vector Flow Imaging
}

\author{
Nguyen, Tin Quoc; Traberg, Marie Sand; Olesen, Jacob Bjerring; Heerwagen, Søren Thorup; Brandt, \\ Andreas Hjelm; Bechsgaard, Thor; Pedersen, Brian Lindegaard; Moshavegh, Ramin; Lönn, Lars; Jensen, \\ Jørgen Arendt \\ Total number of authors:
}

12

Published in:

Ultrasound in Medicine and Biology

Link to article, DOI:

10.1016/j.ultrasmedbio.2020.05.021

Publication date:

2020

Document Version

Peer reviewed version

Link back to DTU Orbit

Citation (APA):

Nguyen, T. Q., Traberg, M. S., Olesen, J. B., Heerwagen, S. T., Brandt, A. H., Bechsgaard, T., Pedersen, B. L., Moshavegh, R., Lönn, L., Jensen, J. A., Nielsen, M. B., \& Hansen, K. L. (2020). Flow Complexity Estimation in Dysfunctional Arteriovenous Dialysis Fistulas using Vector Flow Imaging. Ultrasound in Medicine and Biology, 46(9), 2493-2504. https://doi.org/10.1016/j.ultrasmedbio.2020.05.021

\section{General rights}

Copyright and moral rights for the publications made accessible in the public portal are retained by the authors and/or other copyright owners and it is a condition of accessing publications that users recognise and abide by the legal requirements associated with these rights.

- Users may download and print one copy of any publication from the public portal for the purpose of private study or research.

- You may not further distribute the material or use it for any profit-making activity or commercial gain

- You may freely distribute the URL identifying the publication in the public portal 
2 Flow Complexity Estimation in Dysfunctional Arteriovenous Dialysis Fistulas using Vector Flow Imaging.

\section{Authors}

4 Tin-Quoc Nguyen ${ }^{1,2}$, Marie Sand Traberg ${ }^{5}$, Jacob Bjerring Olesen ${ }^{5,6}$, Søren Thorup Heerwagen ${ }^{1}$, Andreas

5 Hjelm Brandt ${ }^{1}$, Thor Bechsgaard ${ }^{3}$, Brian Lindegaard Pedersen ${ }^{4}$, Ramin Moshavegh ${ }^{5,6}$, Lars Lönn ${ }^{1,2}$, Jørgen

6 Arendt Jensen ${ }^{5}$, Michael Bachmann Nielsen ${ }^{1,2}$, Kristoffer Lindskov Hansen $^{1,2}$

\section{Affiliations}

$8{ }^{1}$ Department of Diagnostic Radiology, Rigshospitalet, Blegdamsvej 9, 2100 Copenhagen, Denmark

$9 \quad{ }^{2}$ Department of Clinical Medicine, University of Copenhagen, Blegdamsvej 3B, 2200 Copenhagen, Denmark

$10{ }^{3}$ Department of Radiology, Odense University Hospital, J.B. Winsløws Vej 4, 5000 Odense C, Denmark

$11{ }^{4}$ Department of Vascular Surgery, Rigshospitalet, Inge Lehmanns Vej 5, 2100 Copenhagen, Denmark

$12{ }^{5}$ Center for Fast Ultrasound Imaging, Department of Health Technology, Technical University of Denmark,

$13 \varnothing$ rsteds Plads building 349, 2800 Lyngby, Denmark

$14{ }^{6}$ BK Medical, Mileparken 34, 2730 Herlev, Denmark

17 Corresponding author: Tin-Quoc Nguyen,

18 Postal address: Græsager 126, 2980 Kokkedal, Denmark,

19 E-mail: tinqnguyen@gmail.com,

Phone: +45 60141546 
Noninvasive assessment is preferred for monitoring arteriovenous dialysis fistula (AVF). Vector concentration assesses flow complexity, which may correlate with stenosis severity. We evaluated if vector concentration could assess stenosis severity in dysfunctional AVF. Vector concentration was estimated in four stenotic phantoms at different pulse repetition frequencies. Spectral Doppler peak velocity and vector concentration were measured in 12 patients with dysfunctional AVF. Additionally, digital subtraction angiography (DSA) was obtained from five patients. In phantoms, vector concentration showed an inverse relationship with stenosis severity and was less affected by aliasing in severe stenoses. In nine stenoses of five patients undergoing DSA, vector concentration correlated strongly with stenosis severity (first stenosis: $r=-0.73, p=0.04$; other stenoses: $r=-0.69, p=0.02$ ) and midstenotic diameter (first stenosis: $r=0.87, p=0.006$; other stenoses: $r=0.70, p=0.02)$ as opposed to peak velocities $(p>0.05)$. Vector concentration is less affected by aliasing in severe stenoses and correlates with DSA in patients with dysfunctional AVF. dialysis fistula, flow complexity, stenosis assessment, patient study, phantom study 


\section{Introduction}

Arteriovenous dialysis fistula (AVF) is the recommended method of vascular access for hemodialysis therapy due to superior long-term patency and reduced risk of infection compared with arteriovenous prosthetic grafts (Allon and Robbin 2002; Huber et al. 2003). However, AVF requires continuous surveillance with Doppler ultrasound or the Ultrasound Dilution Technique to identify stenosis and dysfunction (NKF-KDOQI 2006), and maintenance with percutaneous transluminal angioplasty (PTA) is essential in end-stage renal disease to uphold patency (Aktas et al. 2015; Beathard et al. 2003). AVF patency is commonly assessed with spectral Doppler ultrasound by determining volume flow and resistive index in the feeding artery, while measurements on the venous side remain complicated due to the convoluted course of the veins and the turbulent flow downstream of the anastomosis (Smith et al. 2012; Zamboli et al. 2014).

Vector flow imaging (VFI) is an angle independent ultrasound technique that produces twodimensional velocity vectors (Jensen and Munk 1998). Several in-vivo studies have used VFI to estimate flow velocity and volume flow with high precision compared with magnetic resonance imaging, spectral Doppler ultrasound, and Ultrasound Dilution Technique (Bechsgaard et al. 2017; Brandt et al. 2016; Brandt et al. 2018; Hansen et al. 2014). Flow complexity calculated from VFI images has been associated with differences in the diameters of the carotid artery (Goddi et al. 2018), and a decrease in a VFI-derived parameter for flow complexity called vector concentration has been associated with arterial and aortic valve diseases (Hansen et al. 2017; Hansen et al. 2019a; Saris et al. 2019).

In this proof-of-concept study we investigated the use of vector concentration as a diagnostic tool to distinguish different stenosis severities from one another, as well as a tool for monitoring an effect of a treatment. Vector concentration was estimated in an experimental setup and in-vivo, where scans were performed on stenotic vessel segments. Vector concentration was estimated in four stenotic phantoms at various pulse repetition frequencies (PRF) to determine its robustness to aliasing. Furthermore, vector 
concentration was compared with spectral Doppler peak velocities and digital subtraction angiography (DSA) measurements before and after balloon angioplasty in patients with dysfunctional AVF. The aim was to evaluate if vector concentration could assess stenosis severity and the effect of treatment in dysfunctional AVF by scanning directly on the lesions.

\section{Materials and Methods}

\section{Phantom setup}

Four straight vessel phantoms carrying stenosis of $0 \%, 40 \%, 70 \%$, and $90 \%$ were fabricated in-house. The manufacturing process has been described elsewhere (Lai et al. 2013; Olesen et al. 2018). A flow system (CompuFlow 1000, Shelley Medical Imaging Technologies, Toronto, Canada) induced a pulsating flow pattern for the blood-mimicking fluid (BMF-US, Shelley Medical Imaging Technologies, Toronto, Canada). A 5.5 MHz wide linear transducer (10L2w, BK Medical, Herlev, Denmark) was positioned with the color box for VFI covering the stenosis. VFI data were obtained at 30 frames per second and stored as sequences of 10 seconds on the ultrasound scanner (bk5000, BK Medical, Herlev, Denmark). Presence of aliasing was visually evaluated on the scanner display, and the lowest PRF setting with no noticeable aliasing was defined as the baseline PRF setting for each phantom. Gain was increased to fill the vessel lumen while avoiding blooming artifacts. Wall filtering was automatically set by the scanner. To test repeatability, 10 repeated sequences were obtained at baseline PRF for each of the phantoms. Subsequently, one acquisition was obtained on every PRF setting between $0.2 \mathrm{kHz}$ to $11.9 \mathrm{kHz}$, resulting in an additional 14 sequences of each phantom. 


\section{Study population}

The project was approved by the Danish Data Protection Agency (Journal number 30-1202, ID number 02867). The local Ethics Committee waived approval, since ultrasound scanning of vessels is considered a routine procedure (protocol number: 17030576). Inclusion criteria were patients with a dysfunctional AVF referred for DSA-guided PTA at the Department of Diagnostic Radiology or ultrasound-guided PTA at the Department of Vascular Surgery, Rigshospitalet, Denmark. Informed and written consents were obtained from all participants.

Sixteen patients participated in the study: seven were treated at the Department of Diagnostic Radiology and examined with ultrasound and DSA, while nine patients were treated at the Department of Vascular Surgery using only ultrasound guidance. Four patients were excluded from the study: two occluded AVF had no measurable flow on ultrasound before PTA and rescue was not attempted, one AVF had no measurable flow by ultrasound after PTA, and one patient requested the PTA-procedure to be discontinued. Furthermore, one stenosis defined on DSA was not identified on ultrasound, and two stenoses could not be ultrasound scanned after PTA due to post-procedural bandages obstructing the scan areas. Flowcharts illustrating the patient and stenosis distribution are shown in Fig. 1A and 1B.

\section{In-vivo setup}

Ultrasound scans were performed using a commercial ultrasound scanner (bk5000, BK Medical, Herlev, Denmark) before and after PTA. The color box position and size for VFI were adjusted to cover the region of interest (ROI), ideally fitting a mid-stenotic, pre-stenotic and the adjacent post-stenotic segment. If the stenosis did not fit inside the color box due to its length or out-of-plane geometry, multiple acquisitions were made along the stenosis. PRF was set to the lowest setting with no noticeable aliasing on the scanner display, but if aliasing occurred at the highest setting, i.e. PRF $11.9 \mathrm{kHz}$, VFI data were obtained at this 
setting. Gain was increased to fill the vessel lumen while avoiding blooming artifacts and wall filtering was automatically set by the scanner. A surgical skin-marker was used to indicate the transducer position before PTA, easing reproduction of the transducer position after PTA. One VFI sequence was obtained at each stenosis before and after PTA, respectively. All sequences were obtained at a frame rate of 30 frames per second and contained 10 seconds of VFI data.

All patients were ultrasound scanned within two hours prior to PTA and one hour after PTA except for two post PTA scans, where the patients requested the examinations to be postponed to the following day. A mean vector concentration was calculated from three estimations of each stenosis. Corresponding estimations of peak velocities were obtained with spectral Doppler. Three acquisitions of spectral Doppler peak velocities were obtained at each stenosis, and a mean spectral Doppler peak velocity was calculated for comparison.

\section{Vector flow imaging}

All scans were obtained on a commercially available ultrasound scanner (bk5000, BK Medical, Herlev, Denmark) using a 5.5 MHz wide linear transducer (10L2w, BK Medical, Herlev, Denmark). Flow vectors were visualized within the color box with the direction and magnitude shown by a color wheel. Vector arrows were superimposed onto the velocity field in real time to ease flow interpretation during ultrasound scanning. The obtained VFI data were processed and analyzed offline using an in-house built graphical user interface in MATLAB (MathWorks, Natick, MA, USA) (Moshavegh et al. 2016).

The vector concentration method utilizes the vector angle distribution to derive a parameter for flow complexity (Pedersen et al. 2014). Estimation of vector concentration is performed by marking a ROI and defining a time segment for analysis (Fig. 2). Vector concentration is measured on a scale ranging from 0 to 1, where 1 indicates a completely laminar flow, while an increase in flow complexity decreases the value 
toward 0 . Vector concentration estimation of in-vivo data was performed by splitting the sequence into three partitions of 100 frames. This allowed the ROI to be repositioned to accommodate any operator or patient motion occurring during scans.

\section{Spectral Doppler ultrasound}

Peak velocities were obtained with spectral Doppler ultrasound on the same scanner setup as VFI. The range gate was positioned in the center of the stenosis with the range gate adjusted to cover the central one third of the lumen. Angle correction was manually adjusted to achieve an insonation angle of maximum 60 degrees relative to the direction of blood flow (Fig. 3). PRF was automatically adjusted by the ultrasound scanner, when the baseline was adjusted to fit the spectrograms. Velocities were measured on screenshots of the spectrograms in an offline graphics editor, GNU Image Manipulation Program (The GIMP Development Team, Berkeley, USA).

\section{Angiography}

In an angio-suite (Toshiba Infinix VF-i/SP, Toshiba Medical Systems Corporation, Otawara, Japan), a direct puncture of a superficial adjacent vein in local anesthesia was followed by a placement of a 4 or 5 French short sheath. Guided by fluoroscopy and DSA, iodine contrast (Visipaque $270 \mathrm{mg} / \mathrm{mL}$, GE Healthcare AS, Oslo, Norway) was injected to visualize the vessels. Midstenotic and adjacent nonstenotic diameters of the vessels were measured on the anteroposterior projection of the AVF on a PACS workstation (AGFA Impax, AGFA Technical Imaging Systems, Ridgefield Park, New Jersey) (Fig. 4). Stenosis severity was calculated as (Ota et al. 2005):

$$
\text { Severity }=1-\frac{\text { Midstenotic diameter }}{\text { Nonstenotic diameter }}
$$


157 Austria).

\section{Results}

Phantom model experiment

At baseline PRF, mean vector concentration declined when stenosis severity inclined above $40 \%$, indicating an inverse relationship (Fig. 5, Table 1).

PRF was gradually reduced from $11.9 \mathrm{kHz}$ in each phantom to evaluate the effect of aliasing on vector concentration. This led to an initial visually stronger flow signal that was accompanied by an increase in vector concentration, which declined when aliasing occurred (Fig. 6). The decline in vector concentration due to aliased flow was more pronounced in the $0 \%$ and $40 \%$ stenosis phantoms than the $70 \%$ and $90 \%$ 167 stenosis phantoms.

In-vivo measurements 
midstenotic vessel diameter after PTA $(p=0.0002)$ and a decrease in mean stenosis severity $(p=0.0007)$. This change was accompanied by a significant increase in vector concentration $(p=0.01)$, while no change was found for mean spectral Doppler peak velocities $(p=0.84)$ (Table 2).

Vector concentration was not associated with stenosis severity when including all stenoses $(r=-0.29, p$ $=0.24)$, but showed a strong inverse correlation, when the first stenosis of each AVF was analyzed separately from the second and third stenoses (first stenosis: $r=-0.73, p=0.04$; other stenoses: $r=-0.69, p$ $=0.02$ ). Vector concentration also showed a moderate linear association with midstenotic vessel diameter $(r=0.50, p=0.03)$, which was stronger when the first stenosis was analyzed separately from the second and third stenoses (first stenosis: $r=0.87, p=0.006$; other stenoses: $r=0.70, p=0.02$ ). Spectral Doppler peak velocity for all ultrasound scans $(n=20)$ showed a moderate and inverse linear association with vector concentration $(r=-0.43, p=0.006)$, but was not associated with any DSA measurements (Table 3$)$. Vector concentration and spectral Doppler velocities have been plotted against DSA in Fig. 7.

No linear association was found between change in vector concentration and stenosis severity $(r=-$ $0.57, p=0.11)$, nor between change in vector concentration and midstenotic vessel diameter $(r=0.51, p=$ 0.16). Due to the small sample size, changes were only analyzed as a pool of all stenoses. One outlier amongst the first stenoses was noted to have a large increase in vector concentration (from 0.38 to 0.95 ) compared with other first stenoses. Stenosis severity in this lesion was reduced from $49 \%$ to $0 \%$.

Furthermore, the post PTA ultrasound scan for the outlier was performed on the day after PTA, which may have affected the results. Exclusion of this measurement from the correlation analysis resulted in a significant association between change in vector concentration and midstenotic vessel diameter $(r=0.79, p$ $=0.02$ ), and a tendency for an inverse association between change in vector concentration and stenosis severity $(r=-0.68, p=0.07)$. Change in spectral Doppler peak velocities did not correlate with a change in stenosis severity $(r=-0.11, p=0.78)$ or midstenotic vessel diameter $(r=-0.26, p=0.50)$. The analyses were 
performed on only 9 out of the 10 stenoses due to the absence of one post PTA ultrasound scan. The plots for changes in DSA and ultrasound measurements can be found in Fig. 8.

\section{Discussion}

Vector concentration was not directly associated with the stenosis severity in dysfunctional AVF and only moderately associated with midstenotic diameter. However, when stenoses were analyzed separately in subgroups, the associations became strong. Changes in vector concentration were also associated with changes in midstenotic vessel diameter following PTA with a tendency for an association with stenosis severity. However, these results were based on a limited sample size and a larger study is needed to verify these findings. The current findings support previous studies of vector concentration, where the parameter was used to assess aortic valve stenosis and stenosis of the superior femoral artery (Hansen et al. 2019a; Hansen et al. 2016). Additionally, VFI found increased flow complexity in infants with congenital heart disease, adults with aortic valve stenosis, and adults with carotid stenosis compared with healthy subjects (Hansen et al. 2017; Hansen et al. 2019b; Saris et al. 2019). One study found the presence of complex flow to be associated with the difference in diameter between the common carotid artery and internal carotid sinus (Goddi et al. 2018), further advocating for an association between vector concentration and vessel narrowing.

The absence of an association between vector concentration and stenosis severity when all stenoses were pooled may be explained by how the first stenoses influence flow going toward the second and third stenoses. Fig. 7 illustrates that first stenoses tended to have a lower vector concentration compared with second and third stenoses of similar severity. This indicates that upstream vessel geometry affects flow to downstream stenoses. Similarly, no association was found between changes in measurements before and after PTA until an outlier was excluded from the analyses. The outlier may be explained by the extended delay between PTA and post ultrasound scan as well as the limitation of 2D acquisition performed with 
both VFI and DSA, as out-of-plane movement may conceal essential information about flow and stenosis geometry.

Our spectral Doppler peak velocities correlated moderately with vector concentration, but not with any DSA measurements. Our findings differ from the excellent results reported by Doelman et al., who found midstenotic spectral Doppler peak velocities above $375 \mathrm{~cm} / \mathrm{s}$ to be a strong predictor of a stenosis severity above $50 \%$ regardless of location (Doelman et al. 2005). Meanwhile, our data indicate that the first stenosis in a row tended to have higher peak velocity compared with downstream stenoses of similar stenosis severity. Wo et al. found midstenotic spectral Doppler peak velocities above $500 \mathrm{~cm} / \mathrm{s}$ to be a reliable predictor of a stenosis severity above $50 \%$, but needed to exclude all stenoses at the anastomosis site due to the presence of consistently high peak velocities (Wo et al. 2017). A single peak velocity criterion may not be viable for the whole segment of stenoses. Instead, separate criteria for the different stenosis subgroups are warranted.

Besides measuring peak flow velocities, stenoses may also be identified with other Doppler ultrasound techniques. Spectral broadening can be observed with conventional Doppler ultrasound in turbulent flow and is often present beyond the stenosis (Beach et al. 2010; Corriveau and Johnston 2004; Doelman et al. 2005). However, spectral broadening is routinely not evaluated when assessing stenoses (NKF-KDOQI 2006). VFI obtains additional flow information compared with conventional Doppler ultrasound due to the angle independency. This allows the user to assess flow complexity in the live situation and quantitative estimation could eventually become a supplemental tool to improve flow assessment in the clinic.

VFI is based on conventional pulsed ultrasound emissions and is limited by aliasing similar to conventional pulsed wave Doppler ultrasound. Aliasing occurs at high blood flow velocities, when the Doppler shift exceeds the frequency that can be sampled according to the Nyquist Limit and results in erroneous estimation of velocity and direction (Nelson and Pretorius 1988; Terslev et al. 2017). Aliasing can 
Since vector concentration measures the vector angle distribution within a region of interest, the measure is not only affected by flow complexity, but also by aliasing where vectors are reversed. In this study aliasing was observed directly on the monitor but not analyzed due to lack of quantification tools. Combination of quantified aliasing and vector concentration may lead to a parameter better than vector concentration alone. As indicated in this and previous studies (Hansen et al. 2019a; Hansen et al. 2017), useful vector concentration estimation could potentially be performed in severe stenoses, but will likely be influenced by both flow complexity and aliasing.

Fig. 6 illustrates how the effect of aliasing was different depending on stenosis severity, with the decline being less pronounced in the $70 \%$ and $90 \%$ stenosis phantoms. When PRF was set too high, random "noise vectors" were present in areas with undetected slow flow, which resulted in a wide vector angle distribution, thus a decreased vector concentration. Reducing the PRF from the high setting allowed slower flow to be detected and resulted in an increased signal-to-noise ratio and vector concentration. When aliasing occurred at PRF $5.1 \mathrm{kHz}$ in the $70 \%$ stenosis phantom, vector concentration decreased by $1.0 \%$ compared with baseline, and reducing PRF to $4.1 \mathrm{kHz}$ resulted in a $4.9 \%$ decrease from the baseline value. Similarly, vector concentration decreased by only $1.1 \%$ compared with baseline, when PRF was reduced from $11.9 \mathrm{kHz}$ to $5.1 \mathrm{kHz}$ in the $90 \%$ stenosis phantom. The small changes could indicate that vector (Hansen et al. 2019a; Hansen et al. 2017). VFI data should be obtained at the lowest PRF setting without aliasing whenever possible since vector concentration seems to be affected by aliased vectors. This is also true for spectral Doppler estimation where adjustment of not only PRF, but also spectral baseline, Doppler gain, and angle correction are crucial for correct velocity estimation (Terslev et al. 2017).

Although aliasing may appear to cause problems in stenoses of lower severity, our tentative experiments have shown that VFI is capable of measuring flow velocities up to approximately $250 \mathrm{~cm} / \mathrm{s}$ without aliasing at PRF $11.9 \mathrm{kHz}$. This range is sufficient for measuring some of the fastest blood flows in 
normal physiology (Garcia et al. 2018). Accordingly, blood flow in healthy vessels and low severity stenoses is not expected to be aliased with VFI when used in a clinical setting, and the lower robustness observed in the low severity phantoms may not be relevant, when estimating flow in patients.

The velocity range at a given PRF measured with VFI is limited similar to conventional pulsed Doppler systems. Therefore, simultaneous measurement of fast and slow flow is not possible. Several different methods have been proposed to counteract this limitation (Ekroll et al. 2016; Posada et al. 2016; Saris et al. 2016; Villagómez Hoyos et al. 2016). One of the proposed methods is an adaptive PRF approach that uses unfocused pulse emissions for VFI to simultaneously measure fast and slow flow (Villagómez Hoyos et al. 2016). Some of the shortcomings encountered in stenoses with a large velocity gradient could be alleviated if an adaptive PRF approach was implemented into the presented VFI setup. This could result in a reduction in the amount of noise vectors observed in areas of slow flow and potentially correct the reduction in vector concentration, when the PRF is set inappropriately high.

AVF patency is commonly assessed with spectral Doppler ultrasound or the Ultrasound Dilution Technique (Hansen et al. 2014; Wijnen 2006). While spectral Doppler ultrasound is considered to be an accurate method for detecting arterial stenoses (Zamboli et al. 2014) and categorizing stenosis severity in carotid and renal arteries (Granata et al. 2009; Grant et al. 2003), it did not correlate with any DSA measurements in our study, where midstenotic velocities were measured on the venous side of AVF. Velocity estimation with spectral Doppler ultrasound relies on an assumption of the direction of peak flow. However, the flow angle may have considerable variation near stenoses, and misalignment of the Doppler beam may result in inaccurate velocity estimation (Hatle et al. 1980). Additionally, vessels in AVF are often near parallel to the skin surface, resulting in velocity estimation being performed at near perpendicular flow angles. Adjustment of the Doppler beam to an insonation angle of 60 degrees or less is an often accepted procedure for velocity estimation, but can still result in inaccurate and imprecise measurements (Hoskins 1996; Park et al. 2012). Meanwhile, VFI is an angle independent ultrasound technique that 
eliminates inaccuracies caused by erroneous angle adjustment. The technique determines the flow vectors within the color box and even allows measurements to be performed on flow moving perpendicular to the beam axis. Furthermore, peak velocities that are not located centrally in the lumen (Ford et al. 2008; Kamenskiy et al. 2011) may go unnoticed with conventional spectral Doppler ultrasound, but will be detected with VFI as long as they are in-plane with the ultrasound beam. Alternatively, a 4D/3D ultrasound method has been proposed and found good agreement with DSA in carotid stenosis (Macharzina et al. 2019).

The Ultrasound Dilution Technique is considered the reference standard for flow estimation in AVF, but is limited to being performed during dialysis sessions, thereby prolonging an already cumbersome procedure (Hansen et al. 2014). An additional limitation is that the dilution agent and blood may not mix sufficiently in AVF with slow flow, thus resulting in erroneous volume flow estimation (Hansen et al. 2014; Pavek et al. 1970). Volume flow derived with VFI was reported to have an increased precision and a strong correlation with the Ultrasound Dilution Technique in AVF (Brandt et al. 2016; Hansen et al. 2014), and may be a feasible alternative due to its ease of use.

This study had several limitations. While our results indicate that vector concentration may be used to assess AVF stenoses using separate criteria for first and other stenoses, the study population was limited with only a subgroup undergoing DSA. Furthermore, pre and post PTA results were pooled before correlation analysis due to the small sample size, resulting in the measurements not being independent. A larger study sample is necessary to verify the findings. DSA was obtained in only one plane and all stenoses were assumed to be circular. Likewise, ultrasound measurements were only acquired in 2D and out-ofplane vectors were not obtained. The solution may be a 3D VFI technique, which has been achieved and validated in-vivo with an experimental scanner setup (Holbek et al. 2017). Spectral Doppler peak velocities and VFI were obtained on the venous side of AVF, where flow is known to be turbulent (Zamboli et al. 2014). The combination of turbulence with the tortuous and bulbous geometry of the fistulas complicated 
accurate velocity estimation. We used an in-house built graphic user interface in MATLAB to calculate vector concentration. Even though the method to calculate vector concentration has been described previously (Hansen et al. 2019; Pedersen et al. 2014), the user interface for vector concentration estimation is currently not commercially available. This is a limitation for other research groups who intend to conduct similar studies. Nevertheless, we encourage other researchers to investigate turbulence as a measure for evaluating vessel pathology with ultrasound. Noise could not be omitted from the vector concentration analysis and may have resulted in a decreased estimate when the PRF was set too high. The noise is currently filtered live on the scanner display, and we expect that the noise can be omitted from the analysis in a future build of the user interface. VFI and spectral Doppler peak velocities were obtained by a single immediately after PTA, which may have affected the results.

\section{Conclusion} assessment in dysfunctional AVF.

The vector concentration method had high precision and correlated strongly with DSA measurements, when scanning directly on 10 stenoses in 5 patients with dysfunctional AVF, whereas spectral Doppler peak velocities did not correlate with DSA. However, vector concentration estimation was affected by noise and aliasing artifacts, and a larger study sample is necessary to clarify whether it is a reliable parameter for stenosis severity. This proof-of-concept study demonstrated an approach to perform noninvasive stenosis 
developed and patented the vector flow imaging technique, and receives royalties from the sale of BK

Medical ultrasound scanners with the technique implemented.

\section{References}

Aktas A, Bozkurt A, Aktas B, Kirbas I. Percutaneous transluminal balloon angioplasty in stenosis of native hemodialysis arteriovenous fistulas: technical success and analysis of factors affecting postprocedural fistula patency. Diagn Interv Radiol 2015;21:160-166.

Allon M, Robbin ML. Increasing arteriovenous fistulas in hemodialysis patients: Problems and solutions. Kidney International 2002;62:1109-1124.

Beathard GA, Arnold P, Jackson J, Litchfield T, Physician Operators Forum of RMS Lifeline. Aggressive treatment of early fistula failure. Kidney International 2003;64:1487-1494.

Bechsgaard T, Hansen KL, Brandt AH, Holbek S, Forman JL, Strandberg C, Lönn L, Bækgaard N, Jensen JA, Nielsen MB. Vector and Doppler Ultrasound Velocities Evaluated in a Flow Phantom and the Femoropopliteal Vein. Ultrasound in Medicine \& Biology 2017;43:2477-2487.

Brandt AH, Hansen KL, Ewertsen C, Holbek S, Olesen JB, Moshavegh R, Thomsen C, Jensen JA, Nielsen MB. A Comparison Study of Vector Velocity, Spectral Doppler and Magnetic Resonance of Blood Flow in the Common Carotid Artery. Ultrasound Med Biol 2018;44:1751-1761.

Brandt AH, Jensen J, Hansen KL, Hansen P, Lange T, Rix M, Jensen JA, Lönn L, Nielsen MB. Surveillance for Hemodialysis Access Stenosis: Usefulness of Ultrasound Vector Volume Flow. J Vasc Access 2016;17:483-488.

Ekroll IK, Avdal J, Swillens A, Torp H, Løvstakken L. An Extended Least Squares Method for Aliasing-Resistant Vector Velocity Estimation. IEEE Transactions on Ultrasonics, Ferroelectrics, and Frequency Control 2016;63:1745-1757.

Ford MD, Xie YJ, Wasserman BA, Steinman DA. Is flow in the common carotid artery fully developed? Physiol Meas 2008;29:1335-1349.

Garcia J, Palen RLF van der, Bollache E, Jarvis K, Rose MJ, Barker AJ, Collins JD, Carr JC, Robinson J, Rigsby CK, Markl M. Distribution of blood flow velocity in the normal aorta: Effect of age and gender. Journal of Magnetic Resonance Imaging 2018;47:487-498.

Goddi A, Bortolotto C, Raciti MV, Fiorina I, Aiani L, Magistretti G, Sacchi A, Tinelli C, Calliada F. High-Frame Rate Vector Flow Imaging of the Carotid Bifurcation in Healthy Adults: Comparison With Color Doppler Imaging. Journal of Ultrasound in Medicine 2018;37:2263-2275. 
Granata A, Fiorini F, Andrulli S, Logias F, Gallieni M, Romano G, Sicurezza E, Fiore CE. Doppler ultrasound and renal artery stenosis: An overview. J Ultrasound 2009;12:133-143.

Grant EG, Benson CB, Moneta GL, Alexandrov AV, Baker JD, Bluth El, Carroll BA, Eliasziw M, Gocke J, Hertzberg BS, Katanick S, Needleman L, Pellerito J, Polak JF, Rholl KS, Wooster DL, Zierler RE. Carotid Artery Stenosis: Gray-Scale and Doppler US Diagnosis-Society of Radiologists in Ultrasound Consensus Conference. Radiology 2003;229:340-346.

Hansen K, Hansen PM, Ewertsen C, Lönn L, Jensen J, Nielsen MB. Vector Flow Imaging compared with digital subtraction angiography for stenosis assessment in the superficial femoral artery-a study of vector concentration, velocity ratio and stenosis degree percentage. 2019a;.

Hansen KL, Juul K, Møller-Sørensen H, Nilsson JC, Jensen JA, Nielsen MB. Pediatric Transthoracic Cardiac Vector Flow Imaging - A Preliminary Pictorial Study. Ultrasound Int Open 2019b;5:E20-E26.

Hansen KL, Møller-Sørensen H, Kjaergaard J, Jensen MB, Jensen JA, Nielsen MB. Aortic Valve Stenosis Increases Helical Flow and Flow Complexity: A Study of Intra-Operative Cardiac Vector Flow Imaging. Ultrasound in Medicine \& Biology 2017;43:1607-1617.

Hansen KL, Møller-Sørensen H, Kjaergaard J, Jensen MB, Lund JT, Pedersen MM, Lange T, Jensen JA, Nielsen $\mathrm{MB}$. Intra-Operative Vector Flow Imaging Using Ultrasound of the Ascending Aorta among 40 Patients with Normal, Stenotic and Replaced Aortic Valves. Ultrasound in Medicine \& Biology 2016;42:2414-2422.

Hansen PM, Olesen JB, Pihl MJ, Lange T, Heerwagen S, Pedersen MM, Rix M, Lönn L, Jensen JA, Nielsen MB. Volume Flow in Arteriovenous Fistulas Using Vector Velocity Ultrasound. Ultrasound in Medicine \& Biology 2014;40:2707-2714.

Hatle L, Angelsen BA, Tromsdal A. Non-invasive assessment of aortic stenosis by Doppler ultrasound. Br Heart J 1980;43:284-292.

Holbek S, Hansen KL, Bouzari H, Ewertsen C, Stuart MB, Thomsen C, Nielsen MB, Jensen JA. Common Carotid Artery Flow Measured by 3-D Ultrasonic Vector Flow Imaging and Validated with Magnetic Resonance Imaging. Ultrasound Med Biol 2017;43:2213-2220.

Hoskins PR. Accuracy of maximum velocity estimates made using Doppler ultrasound systems. BJR 1996;69:172-177.

Huber TS, Carter JW, Carter RL, Seeger JM. Patency of autogenous and polytetrafluoroethylene upper extremity arteriovenous hemodialysis accesses: a systematic review. Journal of Vascular Surgery 2003;38:1005-1011.

Jensen JA, Munk P. A new method for estimation of velocity vectors. IEEE Trans Ultrason Ferroelectr Freq Control 1998;45:837-851.

Kamenskiy AV, Dzenis YA, MacTaggart JN, Desyatova AS, Pipinos II. In vivo three-dimensional blood velocity profile shapes in the human common, internal, and external carotid arteries. Journal of Vascular Surgery 2011;54:1011-1020. 
Lai SSM, Yiu BYS, Poon AKK, Yu ACH. Design of Anthropomorphic Flow Phantoms Based on Rapid Prototyping of Compliant Vessel Geometries. Ultrasound in Medicine \& Biology 2013;39:16541664.

Macharzina RR, Kocher S, Messe SR, Kammerer T, Hoffmann F, Vogt M, Vach W, Fan N, Rastan A, Neumann F-J, Zeller T. Improved Carotid Stenosis Quantification on Novel 4D/3D-Doppler Ultrasonography Indexing to the Common Carotid Artery. Ultraschall in Med 2019 [cited 2019 Sep 2]; Available from: http://www.thieme-connect.de/DOI/DOI?10.1055/a-0628-6459

Moshavegh R, Martins B, Hansen K, Bechsgaard T, Nielsen MB, Jensen J. Hybrid segmentation of vessels and automated flow measures in in-vivo ultrasound imaging. 2016. pp. 1-4.

Nelson TR, Pretorius DH. The Doppler signal: where does it come from and what does it mean? AJR Am J Roentgenol 1988;151:439-447.

NKF-KDOQI. Clinical Practice Guidelines for Vascular Access. American Journal of Kidney Diseases 2006;48:S176-S247.

Olesen JB, Villagomez-Hoyos CA, Moller ND, Ewertsen C, Hansen KL, Nielsen MB, Bech B, Lonn L, Traberg MS, Jensen JA. Noninvasive Estimation of Pressure Changes Using 2-D Vector Velocity Ultrasound: An Experimental Study With In Vivo Examples. IEEE Trans Ultrason Ferroelectr Freq Control 2018;65:709-719.

Ota H, Takase K, Rikimaru H, Tsuboi M, Yamada T, Sato A, Higano S, Ishibashi T, Takahashi S. Quantitative Vascular Measurements in Arterial Occlusive Disease. RadioGraphics 2005;25:1141-1158.

Park MY, Jung SE, Byun JY, Kim JH, Joo GE. Effect of Beam-Flow Angle on Velocity Measurements in Modern Doppler Ultrasound Systems. American Journal of Roentgenology 2012;198:1139-1143.

Pavek E, Pavek K, Boska D. Mixing and observation errors in indicator-dilution studies. Journal of Applied Physiology 1970;28:733-740.

Pedersen MM, Pihl MJ, Haugaard P, Hansen KL, Lange T, Lönn L, Nielsen MB, Jensen JA. Novel flow quantification of the carotid bulb and the common carotid artery with vector flow ultrasound. Ultrasound Med Biol 2014;40:2700-2706.

Posada D, Porée J, Pellissier A, Chayer B, Tournoux F, Cloutier G, Garcia D. Staggered Multiple-PRF Ultrafast Color Doppler. IEEE Transactions on Medical Imaging 2016;35:1510-1521.

Saris AECM, Hansen HHG, Fekkes S, Menssen J, Nillesen MM, de Korte CL. In Vivo Blood Velocity Vector Imaging Using Adaptive Velocity Compounding in the Carotid Artery Bifurcation. Ultrasound Med Biol 2019;45:1691-1707.

Saris AECM, Hansen HHG, Fekkes S, Nillesen MM, Rutten MCM, de Korte CL. A Comparison Between Compounding Techniques Using Large Beam-Steered Plane Wave Imaging for Blood Vector Velocity Imaging in a Carotid Artery Model. IEEE Transactions on Ultrasonics, Ferroelectrics, and Frequency Control 2016;63:1758-1771.

Smith GE, Gohil R, Chetter IC. Factors affecting the patency of arteriovenous fistulas for dialysis access. Journal of Vascular Surgery 2012;55:849-855. 
Terslev L, Diamantopoulos AP, Døhn UM, Schmidt WA, Torp-Pedersen S. Settings and artefacts relevant for Doppler ultrasound in large vessel vasculitis. Arthritis Res Ther 2017;19:1-10.

Villagómez Hoyos CA, Stuart MB, Hansen KL, Nielsen MB, Jensen JA. Accurate Angle Estimator for HighFrame-Rate 2-D Vector Flow Imaging. IEEE Transactions on Ultrasonics, Ferroelectrics, and Frequency Control 2016;63:842-853.

Wijnen E. Comparison between two on-line reversed line position hemodialysis vascular access flow measurement techniques: saline dilution and thermodilution. ASAIO J 2006;52:410-415.

Zamboli P, Fiorini F, D’Amelio A, Fatuzzo P, Granata A. Color Doppler ultrasound and arteriovenous fistulas for hemodialysis. J Ultrasound 2014;17:253-263.

\section{Figure captions}

Fig. 1: A) Overview of the patients in the study, " $n$ " represents the number of patients. B) Overview of the stenoses in the study, " $N$ " represents the number of stenoses. "Pre PTA" and "Post PTA" refer to examinations performed before and after PTA. The first stenosis in an AVF was referred as "First stenosis", while any second and third stenoses were pooled as "Other stenoses". SDUS = Spectral Doppler ultrasound

Fig. 2: Vector concentration estimation in a $40 \%(A)$ and $70 \%(B)$ stenosis phantom. The two examples were examined with a linear transducer at PRF $2.6 \mathrm{kHz}$ and $6.9 \mathrm{kHz}$, respectively. The red box delineates the ROI for vector concentration estimation.

Fig. 3: A) Velocity estimation with Spectral Doppler ultrasound of the first stenosis ( $\left.{ }^{*}\right)$ in an AVF with three stenoses. B) VFI of the same stenosis. Small areas of aliasing can be seen in the midstenotic flow. Stenosis severity was determined to be $47 \%$ on DSA. 

Fig. 4: Routine anteroposterior DSA in one plane before (A) and after (B) balloon angioplasty of the same AVF as illustrated in Fig. 3. Stenosis severity of the three lesions (marked 1-3) was reduced from 47\%, 63\%, and $66 \%$ to $35 \%, 43 \%$, and $29 \%$ after balloon angioplasty, respectively.

Fig. 5: Mean midstenotic vector concentration measured in four stenotic phantom models of $0 \%, 40 \%, 70 \%$, and $90 \%$ constriction.

Fig. 6: Dot plots illustrating the effect of adjusting PRF on vector concentration in four stenotic phantoms. The lowest PRF for each stenosis phantom that presented no aliasing was defined as the baseline PRF.

Fig. 7: Vector concentration and spectral Doppler peak velocities plotted against stenosis severity and midstenotic vessel diameter measured on DSA. Pre- and post-PTA results were pooled. The solid line illustrates the best linear fit for the first stenoses, while the dotted line is the best linear fit for the second and third stenoses. The open and filled symbols indicate if flow was nonaliased or aliased, respectively.

Fig. 8: In-vivo results of the change in vector concentration and spectral Doppler peak velocity plotted against change in stenosis severity and midstenotic vessel diameter following PTA.

\section{Tables}

\begin{tabular}{|lcc|}
\hline Stenosis severity & PRF (kHz) & Mean VC (SD) \\
\hline $0 \%$ & 2.6 & $0.95(0.01)$ \\
\hline $40 \%$ & 2.6 & $0.95(0.02)$ \\
\hline $70 \%$ & 6.9 & $0.81(0.02)$ \\
\hline
\end{tabular}




\begin{tabular}{|lll|}
\hline $90 \%$ & 11.9 & $0.64(0.02483$ \\
\hline
\end{tabular}

487 Table 1: Baseline PRF settings and mean vector concentration for 10 repeated measurements. Baseline PRF 488 was defined as the lowest PRF setting that displayed flow without aliasing. VC = Vector concentration. SD = 489 Standard deviation.

\begin{tabular}{|lllll|}
\hline & Before PTA (SD) & After PTA (SD) & Mean difference [Cl95] & $p$-value \\
\hline Mean vector concentration & $0.70(0.24)$ & $0.80(0.17)$ & $+0.13[0.03 ; 0.22]$ & 0.01 \\
\hline Mean peak SDUS (cm/s) & $305.0(130.1)$ & $333.1(112.6)$ & $+5.75[-54.2 ; 65.7]$ & 0.84 \\
\hline Mean stenosis severity (\%) & $62(16)$ & $24(14)$ & $-0.37[-0.54 ;-0.21]$ & 0.0007 \\
\hline Mean vessel diameter (cm) & $0.23(0.13)$ & $0.49(0.08)$ & $+0.25[0.14 ; 0.34]$ & 0.0002 \\
\hline
\end{tabular}

Table 2: Mean values and mean differences calculated with paired t-tests before and after PTA. Mean

492 for all patients $(n=12)$. Mean difference of stenosis severity and midstenotic vessel diameter were 493 calculated for 5 patients. SD = Standard deviation. CI95 =95\% confidence interval. 


\begin{tabular}{|lll|}
\hline & Vector concentration & Spectral Doppler velocity \\
\hline Stenosis severity, all stenoses & $r=-0.29, p=0.24$ & $r=-0.11, p=0.66$ \\
\hline Stenosis severity, first stenosis & $r=-0.73, p=0.04$ & $r=-0.16, p=0.70$ \\
\hline Stenosis severity, other stenoses & $r=-0.69, p=0.02$ & $r=0.10, p=0.77$ \\
\hline Vessel diameter, all stenoses & $r=0.50, p=0.03$ & $r=-0.25, p=0.30$ \\
\hline Vessel diameter, first stenosis & $r=0.87, p=0.006$ & $r=-0.53, p=0.17$ \\
\hline Vessel diameter, other stenoses & $r=0.70, p=0.02$ & $r=-0.26, p=0.44$ \\
\hline
\end{tabular}

Table 3: Pearson correlation coefficients, $r$, for in-vivo ultrasound and DSA measurements. 